\title{
Introduction to the Validation of a Dissolution Apparatus
}

\author{
Sharon M. Averell Frost \\ Senior Technical Services Scientist, Technical Services Dept., \\ Wyeth Vaccines, 4300 Oak Park, Sanford, NC, 27330
}

\section{Introduction}

Dissolution is defined as the process by which a known amount of drug substance goes into solution per unit of time under standardized conditions. The primary goal of dissolution testing is to be used as a qualitative tool to provide measurements of the bioavailability of a drug as well as to demonstrate bioequivalence from batch-to-batch. The bioavailability and bioequivalence data obtained as a result of dissolution testing can be used to guide the development of a new formulation and product development processes toward product optimization, as well as to ensure continuing product quality and performance of the manufacturing process. In addition, dissolution is a requirement for regulatory approval for product marketing and is a vital component of the overall quality control program (1).

Dissolution testing is conducted using a dissolution apparatus that conforms to the specifications outlined in the United States Pharmacopeia. There are seven types of dissolution apparatus; the apparatus chosen to perform dissolution testing depends primarily on the drug dosage form.

In order to have a high degree of assurance that the dissolution apparatus is consistent and accurate in its performance, validation is required. Validation is defined as documented evidence that provides a high degree of assurance that a specific instrument performs according to manufacturer's specifications and user requirements. Validation is achieved by performing a series of validation activities; for a dissolution apparatus, validation is obtained through installation qualification and operational qualification.

\section{Installation Qualification}

Installation qualification consists of documented verification that all key aspects of the dissolution apparatus are in working condition and have been properly installed in accordance with manufacturer's specifications in the proper operating environment. The installation qualification of a dissolution apparatus should include the following verifications:

\section{- Preventive maintenance}

The installation qualification should document that the dissolution apparatus is enrolled in a preventive maintenance program to assure that the system continues to operate properly and no component part of the system becomes inoperable due to wear and use.

\section{- Calibration}

The installation qualification should document that specific devices contained within the dissolution appa-
Email:frosts1@wyeth.com

ratus (e.g., speed, time, and temperature displays) have been calibrated to traceable standards.

Documentation should include the date calibration was performed and when calibration is due.

\section{- SOPs}

The installation qualification should document that all SOPs pertaining to the dissolution apparatus are approved and in place. Applicable SOPs may include preventive maintenance, calibration, operation, document archival, and equipment logbook usage.

\section{- Utilities}

The installation qualification should document the manufacturer's specifications for required utilities and verify that the appropriate utilities are available for the system. For example, utility verification may include confirming that the appropriate electrical power requirements (voltage, amperage, safety cut-offs) are documented and comply with manufacturer's specifications.

\section{- Computerized System}

If the dissolution apparatus is computerized, the installation qualification should document the manufacturer's specifications for the computer system and verify that the computer system in place complies with manufacturer's specifications. Documentation should include model and serial number of associated hardware, operating system name and version, software name and version, location of master and back-up files, and CPU requirements such as speed and hard drive capacity.

\section{- Environmental Conditions}

The installation qualification should document the environmental conditions that surround the dissolution apparatus conform to USP standards, which require that no part of the assembly, including the environment in which the assembly is placed, contributes significant motion, agitation, or vibration beyond that due to the smoothly rotating stirring element.

\section{- Equipment Information}

The installation qualification should document equipment information including the manufacturer, model number, and serial number of the dissolution apparatus, and verify that the information complies with purchase orders and user requirements. In addition, verification of the unit's compliance with requirements outlined by the USP < 711 > Dissolution General Chapter, and specific to each dissolution apparatus, should be 
performed. Requirements to be verified are summarized below (2)

\section{- Apparatus 1}

- Vessel: cylindrical, 160-210 mm high, inside diameter $98-106 \mathrm{~mm}$, nominal capacity is $1000 \mathrm{~mL}$; sides are flanged at the top.

- Shaft: positioned so that its axis is not more than $2 \mathrm{~mm}$ at any point from the vertical axis of the vessel and rotates smoothly and without significant wobble.

- Materials of Construction: Shaft and basket components are stainless steel, type 316 or equivalent.

- Basket position: the distance between the inside bottom of the vessel and the basket is maintained at $25+/-2 \mathrm{~mm}$ during the test.

\section{- Apparatus 2}

- Vessel: cylindrical, 160-210 mm high, inside diameter $98-106 \mathrm{~mm}$, nominal capacity is $1000 \mathrm{~mL}$; sides are flanged at the top.

- Shaft: positioned so that its axis is not more than $2 \mathrm{~mm}$ at any point from the vertical axis of the vessel and rotates smoothly and without significant wobble.

- Materials of Construction: Shaft and blade are a single entity that may be coated with a suitable inert coating.

- Blade position: the distance between the inside bottom of the vessel and the blade is maintained at $25+/-2 \mathrm{~mm}$ during the test. The blade passes through the diameter of the shaft so that the bottom of the blade is flush with the bottom of the shaft.

\section{- Apparatus 3}

- Reciprocating Cylinder: positioned so that during the upward and downward stroke, the reciprocating cylinder moves through a total distance of 9.9- $10.1 \mathrm{~cm}$.

- Materials of Construction: Fittings are stainless steel, type 316 or equivalent.

\section{- Apparatus 4}

- Materials of Construction: Flow-through cell, composed of transparent and inert material, is mounted vertically with a filter system that prevents escape of undissolved particles from the top of the cell. Tube connections are of polytef tubing with $1.6-\mathrm{mm}$ diameter and chemically inert flanged-end connections.

- Cell Assembly: Cell diameters are 12 and 22.6 $\mathrm{mm}$; the apparatus uses a clamp mechanism and two O-rings for the fixation of the cell assembly.

- Apparatus 5

- Vessel: cylindrical, 160-210 mm high, inside diameter $98-106 \mathrm{~mm}$, nominal capacity is $1000 \mathrm{~mL}$; sides are flanged at the top.

- Shaft: positioned so that its axis is not more than $2 \mathrm{~mm}$ at any point from the vertical axis of the vessel and rotates smoothly and without significant wobble.

- Materials of Construction: Shaft and blade are a single entity that may be coated with a suitable inert coating. Disk assembly is stainless steel.

- Blade Position: the distance between the surface of the disk assembly and the blade is maintained at $25+/-2 \mathrm{~mm}$ during the test. The disk assembly holds the system flat and is positioned such that the release surface is parallel with the bottom of the paddle blade.

\section{- Apparatus 6}

- Vessel: cylindrical, 160-210 mm high, inside diameter $98-106 \mathrm{~mm}$, nominal capacity is $1000 \mathrm{~mL}$; sides are flanged at the top.

- Shaft: positioned so that its axis is not more than $2 \mathrm{~mm}$ at any point from the vertical axis of the vessel and rotates smoothly and without significant wobble.

- Materials of Construction: Cylinder stirring element is stainless steel, type 316 or equivalent.

- Cylinder Position: the distance between the inside bottom of the vessel and the cylinder is maintained at $25+/-2 \mathrm{~mm}$ during the test.

\section{Operational Qualification}

Operational Qualification consists of documented evidence that the equipment operates as intended and is capable of consistent operation within established specifications. The operational qualification of a dissolution apparatus should include the following verifications:

\section{- System Suitability (Calibration)}

A system suitability test using USP calibrators should be conducted during operational qualification testing. The procedure for dissolution and sampling is outlined in the Certificates supplied with each USP Calibrator tablet for each apparatus. The calibrators used for the test are disintegrating tablets (Prednisone) and nondisintegrating tablets (Salicylic Acid). The test is considered successful if the percent of drug released at 30 minutes falls within a pre-established range. The ranges for each combination of apparatus and calibrators at 50 or 100 RPM are established by the USP and are different for each lot of calibrators. This test must be conducted for each of the vessels contained within a dissolution apparatus. For a vessel to be acceptable, it must, individually, provide acceptable drug release from the calibrator (3). For sample aliquots withdrawn from the vessels, the solutions are analyzed using previously validated HPLC or UV/Vis methods (depending on the monograph) that yield acceptable peak resolution and elution time. The analysis can then be used to create a profile of percent drug released vs. time.

- Validation of Automated Versus Manual Procedures At a minimum, the following verifications should be 
included in the operational qualification of an automated dissolution system (4):

\section{- Software/hardware Communication} Verify that the software accurately controls the hardware.

\section{- Operator Interface Functions}

Verify that the system can be accurately controlled through functions pre-defined by the manufacturer. For example, operator interface testing may include confirming that all menu options are available and that a file can be successfully created and modified.

- Stress/boundary/challenge Testing Verify that the system successfully performs under stress and/or challenge conditions that may be present during operation. For example, stress/boundary/challenge testing may include confirming that a maximum number of files can be processed simultaneously, or that the system can perform simultaneous functions.

In addition, if the dissolution apparatus employs a computerized system, 21 CFR Part 11 requirements must be considered. At a minimum, the following verifications should be included in the operational qualification:

- Report Printing/handling

Verify that all reports defined by the manufacturer are available and accurate.

- Audit Trail

Verify that the audit trail accurately captures changes to the system, including file creation, modification, deletion, and data processing.

- Electronic Signatures

Verify that electronic signatures are operational and accurate.

- Data archival and restoration

Verify that data is successfully archived and restored.

- Data Integrity Verify that data integrity is not compromised during data backup, archival, or restoration functions.

- System Security Verify that all applicable system security measures are operational (e.g., user name, password, screen saver, automatic time-outs, user level definitions, etc.)

\section{- Validation of Analytical Methods Employed in} Quantitative Analysis of Dissolution Samples Analytical methods used to perform analysis of dissolution should be validated. Generally, this method validation is not part of the operational qualification of the dissolution apparatus. However, validated methods should be employed to analyze the samples withdrawn as part of the system suitability testing performed during the operational qualification. (5)

\section{- Temperature Distribution Study}

A temperature distribution study should be conducted during the operational qualification. The study should include temperature mapping of each vessel contained within the dissolution apparatus. Temperature should be mapped using a data acquisition system for a minimum time that is based on the monograph or 1 hour, whichever is greater. The temperature of Apparatus $1,2,3$, and 4 must remain at $37^{\circ} \mathrm{C} \pm 0.5^{\circ} \mathrm{C}$; the temperature of Apparatus 5 and 6 must remain at $32^{\circ} \mathrm{C} \pm 0.5^{\circ} \mathrm{C}$.

\section{- Rotation Speed Study}

A rotation speed study should be conducted during the operational qualification. The study should include a measurement of the speed of the shaft rotation for each vessel contained within the dissolution apparatus. Speed should be measured using a photo tachometer for 30 minutes or the time specified in the individual monograph, whichever is greater. The speed of the shaft rotation should be verified to be within $4 \%$ of the speed specified in the monograph.

\section{Conclusion}

At the conclusion of acceptable installation and operational qualifications, the dissolution apparatus is considered validated and acceptable for use to perform dissolution testing. The system suitability tests should be performed after any significant equipment change (e.g., a change from a basket apparatus to a paddle apparatus, unless multiple apparatus are qualified at the time of validation) or relocation of the dissolution apparatus (e.g., to another laboratory). Barring any significant change, the system suitability tests should be conducted at least twice a year as part of a robust preventive maintenance program.

\section{References}

1. Ansel, Howard C., Lloyd V. Allen, Jr., and Nicholas G. Popovich. Pharmaceutical Dosage Forms and Drug Delivery Systems. Baltimore, Maryland: Lippincott Williams \& Wilkins, 7th edition, 1999.

2. United States Pharmacopeial Convention, Inc. United States Pharmacopeia 26. Rockville, Maryland: United States Pharmacopeial Convention, Inc. 2003.

3. Qureshi, S.A. "The USP Dissolution Apparatus Suitability Test." Drug Information Journal. 1996; 30; 1055-1061.

4. GAMP Guide Forum. GAMP 4. GAMP Guide Forum and ISPE. 2003.

5. Food and Drug Administration. Guidance for Industry, Dissolution Testing of Immediate Release Solid Oral Dosage Forms. Rockville, Maryland: Food and Drug Administration, Center for Drug Evaluation and Research. 1997. 